\section{Polarisation dependence of two-photon absorption generated by band-limited amplified spontaneous emission noise in silicon avalanche photodiodes}

J.A. O’Dowd, W.H. Guo, E. Flood, M. Lynch, A.L. Bradley, D.C. Kilper and J.F. Donegan

Two-photon absorption (TPA) generated by unpolarised band-limited amplified spontaneous emission (BL-ASE) noise is found to be within $5 \%$ of that generated by circularly polarised BL-ASE noise for silicon-based devices. A simple formula that describes the TPA generated by partially polarised BL-ASE noise as a function of material dichroism is given and verified experimentally.

Introduction: Two-photon absorption (TPA), owing to its sensitivity to the peak power of modulated signals, has recently attracted interest as a means of carrying out network impairment monitoring and may find applications in photonic integrated circuits [1]. The TPA generated photocurrent has been used to monitor impairments such as chromatic dispersion [2], and polarisation mode dispersion [3]. Monitoring the change in the optical-signal-to-noise-ratio (OSNR) related to the increased level of amplified spontaneous emission (ASE), which results from the re-amplification of a signal using optical amplifiers, is also useful [3]. Of particular importance are measurements of OSNR within the channel bandwidth (in-band), which are not reliably carried out using spectral analysis [4]. Monitoring of in-band OSNR using TPA was analysed in [1] and implemented in [5] and [6]. In [3] the level of TPA generated by band-limited ASE (BL-ASE) noise was incorrectly assumed to be equal to that generated by a continuous-wave (CW) signal with the same average power. This assumption was corrected in [7] where the TPA generated by polarised BL-ASE noise was found to be twice that generated by a CW signal when both have the same polarisation and the same average power. However, the amount of TPA generated when the BL-ASE noise is unpolarised or partially polarised has not previously been shown. In this Letter, we investigate the level of TPA generated by BL-ASE noise for the most general cases, i.e. where the BL-ASE noise is completely polarised, completely unpolarised or partially polarised. We introduce a formula that describes the dependence of TPA generated by partially polarised BL-ASE noise on both the degree of polarisation and the residual polarisation state of the BL-ASE noise. Two special cases in which the residual polarisation is either linear or circular are investigated both theoretically and experimentally. It is found that the level of TPA generated by unpolarised ASE is close to that generated by circularly polarised ASE noise where both have the same average power.

Theory: The TPA current generated in silicon (Si) at room temperature for incident light along the [001] crystallographic direction has previously been described in [8] as:

$$
i_{T P A}=C\left(\left\langle I^{2}\right\rangle-\delta\left\langle S_{3}^{2}\right\rangle\right)
$$

where $S_{i}$ is a Stokes variable (for $i=1,2,3$ ), \langle\rangle indicates a time average, $\left\langle S_{i}\right\rangle$ is a Stokes parameter (for $i=1,2,3$ ), $I$ is the input light intensity, $C$ is a polarisation independent absorption coefficient, $\delta$ is the dichroism parameter, which has previously been reported for $\mathrm{Si}$ in the region of $1550 \mathrm{~nm}$ as being approximately +0.37 at room temperature [8]. $\left\langle I^{2}\right\rangle$ and $\left\langle S_{3}^{2}\right\rangle$ can be difficult to measure and therefore one would like to express this in terms of the corresponding mean quantities and the degree of polarisation. The degree of polarisation $D$ can be defined as $D=\left(\sqrt{\left\langle S_{1}\right\rangle^{2}+\left\langle S_{2}\right\rangle^{2}+\left\langle S_{3}\right\rangle^{2}}\right) /\langle I\rangle$, with $0 \leq D \leq 1$ where $D=1$ and $D=0$ correspond to completely polarised and completely unpolarised, respectively. The residual polarisation of partially polarised light is defined by $\left\langle S_{1}\right\rangle,\left\langle S_{2}\right\rangle$, and $\left\langle S_{3}\right\rangle$, which are experimentally measurable as well as the light intensity $\langle I\rangle$. At any instant in time for a signal which is random in the time domain the light is fully polarised; therefore there is always a relationship between the three Stokes variables and the light intensity which can be expressed as $I^{2}=S_{1}^{2}+S_{2}^{2}+S_{3}^{2}$ for each point in time [9]. To calculate the dependence of $i_{T P A}$ on the degree and state of polarisation, $\left\langle S_{3}^{2}\right\rangle$ must be expressed in terms of $\langle I\rangle$ and $\left\langle S_{i}\right\rangle(i=1,2,3)$ as shown in (1). In [9] the individual marginal probability density function for each of the Stokes variables is found for partially polarised light for correlated Gaussian fields. The moments of the
Stokes variables $\left\langle S_{i}^{n}\right\rangle$ are also derived (where $n$ is any positive integer) and expressed in terms of $I$ and the three Stokes parameters. We adopt this formalism for $n=2$ :

$$
\left\langle S_{i}^{2}\right\rangle=\frac{\langle I\rangle^{2}-\left\langle S_{j}\right\rangle^{2}-\left\langle S_{k}\right\rangle^{2}+3\left\langle S_{i}\right\rangle^{2}}{2}
$$

where $i, j, k=1,2,3$ and $i \neq j \neq k$. By substituting (2) into (1) the TPA generated by partially polarised BL-ASE noise can be described in terms of experimentally measurable parameters:

$$
i_{T P A}=C\left[\frac{3-\delta}{2}\langle I\rangle^{2}+\frac{1+\delta}{2}\left(\left\langle S_{1}\right\rangle^{2}+\left\langle S_{2}\right\rangle^{2}\right)+\frac{1-3 \delta}{2}\left\langle S_{3}\right\rangle^{2}\right]
$$

By introducing the definition of the degree of polarisation $D$ (3) can be expressed as:

$$
i_{T P A}=C\left[\frac{3-\delta+(1+\delta) D^{2}}{2}\langle I\rangle^{2}-2 \delta\left\langle S_{3}\right\rangle^{2}\right]
$$

The TPA generated by partially polarised BL-ASE noise for two special cases where the residual polarisation is linear and circular, respectively, are considered, although (4) can be used to describe TPA generated by partially polarised BL-ASE noise with any state of residual polarisation. First, for linear residual polarisation $\left\langle S_{3}\right\rangle=0$ and $\left\langle S_{1}\right\rangle^{2}+\left\langle S_{2}\right\rangle^{2}=\langle I\rangle^{2} D^{2}$, which allows the TPA generated by such a signal to be expressed using (4) as:

$$
i_{T P A}=C\langle I\rangle^{2}\left(\frac{3-\delta}{2}+\frac{1+\delta}{2} D^{2}\right)
$$

Secondly, for circular residual polarisation $\left\langle S_{1}\right\rangle=\left\langle S_{2}\right\rangle=0$ and $\left\langle S_{3}\right\rangle^{2}=\langle I\rangle^{2} D^{2}$ allowing the TPA generated to be expressed using (4) as:

$$
i_{T P A}=C\langle I\rangle^{2}\left(\frac{3-\delta}{2}+\frac{1-3 \delta}{2} D^{2}\right)
$$

As is described by (6), the amount of TPA resulting from circularly polarised BL-ASE noise can be less than that generated by depolarised BL-ASE noise, with this relationship being governed by $\delta$.

Experiment: TPA measurements were carried out using a commercially available (Perkin Elmer) Si avalanche photodiode (APD), which allows detection of TPA at low input optical powers. A BL-ASE noise signal was created by passing the output of an erbium-doped fibre amplifier (EDFA) with no optical input through a $1 \mathrm{~nm}$ bandpass filter, see Fig. 1. The BL-ASE noise signal was re-amplified using a second EDFA and then refiltered using another $1 \mathrm{~nm}$ bandpass filter to remove any ASE outside the $1 \mathrm{~nm}$ passband. The BL-ASE noise was separated into two paths using a 50:50 coupler. One path (polarised) was passed through a polariser. The second path (unpolarised) bypassed the polariser. Both paths had variable optical attenuators that allowed $D$ to be set by varying the ratio of the transmitted power for both arms. Power meters were used to monitor the polarised and total average powers, respectively, which in turn allowed the depolarised power and $D$ to be calculated. The polarisation of the signal in the polarised arm was then set using a polarisation controller. The signal was launched into free space where both its polarisation and its power were monitored using a free space power meter and free space polarisation analyser, respectively. The signal passed through a chopper before being focused onto the Si-APD using a $10 \times$ microscope objective. The TPA generated photocurrent was recorded using a lock-in amplifier.

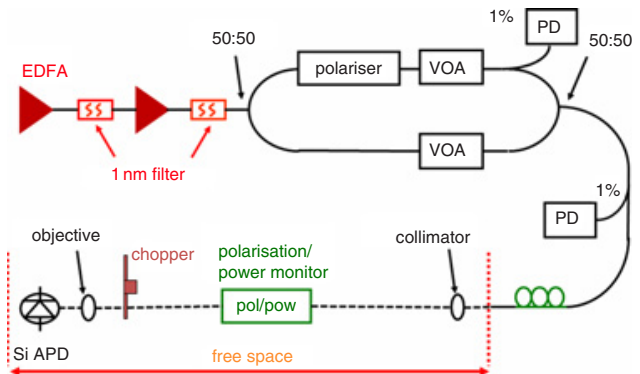

Fig. 1 Schematic diagram of experimental setup VOA: variable optical attenuator; Si-APD: silicon avalanche photodiode; PD average power monitor 
The dependence of the TPA generated photocurrent on $D$ was investigated. The average power on the detector was kept constant at $1.94 \mathrm{~mW}$, which was high enough to ensure that TPA dominated over single photon absorption in the APD. The TPA generated photocurrent was recorded as a function of $D$ for BL-ASE noise with the residual polarisation set to be linearly and then circularly polarised, respectively, see Fig. 2. The experimental results for linearly and circularly-polarised residual polarisation are fitted using (5) and (6), respectively, with the resultant fits shown to agree very well with the experimental data. It can be seen in Fig. 2 that the level of TPA generated by unpolarised BL-ASE noise is very close to that generated by circularly polarised BL-ASE noise (just $\sim 5 \%$ more) but significantly less than that generated by linearly polarised BL-ASE noise ( $\sim 34 \%$ less $)$, as expected based on (1) and (2).

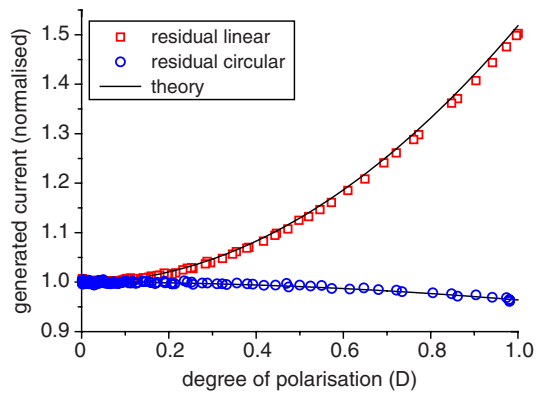

Fig. 2 Dependence of TPA generated photocurrent on degree of polarisation (D) for band-limited amplified spontaneous emission (BL-ASE) noise with residual linear and residual circular polarisations, respectively

TPA signal normalised such that TPA generated by depolarised BL-ASE is equal to 1

Conclusion: The level of TPA generated by unpolarised BL-ASE noise was found to be within $5 \%$ of that generated by circularly polarised BLASE noise for silicon with a dichroism parameter of +0.37 . A simple formula was given, which describes the TPA generated by partially polarised BL-ASE noise for any state of residual polarisation and degree of polarisation as a function of the material dichroism. The theory is shown to match the measured response of a silicon avalanche photodiode device.

Acknowledgment: This work was supported by Science Foundation Ireland under its CSET, Centre for Telecommunications Value-chain Research (CTVR) grant number 08/CE/I1523.
(C) The Institution of Engineering and Technology 2011

24 October 2011

doi: $10.1049 /$ el.2011.3317

One or more of the Figures in this Letter are available in colour online.

J.A. O'Dowd, W.H. Guo, E. Flood, M. Lynch, A.L. Bradley and J.F. Donegan (CTVR, Semiconductor Photonics Group, School of Physics, Trinity College Dublin, Ireland)

E-mail: jodowd@tcd.ie

D.C. Kilper (Bell Laboratories, Alcatel-Lucent, Holmdel, NJ 07733 , USA)

J.A. O’Dowd: Now with Intune Networks, Park West Business Park, Dublin, Ireland

\section{References}

1 Kilper, D.C.: 'Nonlinear optical techniques for OPM', in Chen, C.C.K. (Ed.): 'Optical performance monitoring techniques for next generation photonic networks' (Elsevier, 2010), pp. 301-317

2 Bondarczuk, K., Maguire, P.J., Reid, D., Barry, L.P., O’Dowd, J., Guo, W.H., Lynch, M., Bradley, A.L., and Donegan, J.F.: 'Chromatic dispersion monitoring for high-speed WDM systems using two-photon absorption in a semiconductor microcavity', IEEE J. Quantum Electron., 2009, 45, (1), pp. 90-99

3 Wielandy, S., Fishteyn, M., and Zhu, B.: 'Optical performance monitoring using nonlinear detection', J. Lightwave Technol., 2004, 22, (3), pp. 784-793

4 Kilper, D.C., and Weingartner, W.: 'Monitoring optical network performance degradation due to amplifier noise', J. Lightwave Technol., 2003, 21, (5), pp. 1171-1178

5 Dinu, M., Kilper, D.C., and Stuart, H.R.: 'Optical performance monitoring using data stream intensity autocorrelation', J. Lightwave Technol., 2006, 24, (3), pp. 1194-1202

6 Reid, D.A., Bondarczuk, K., Dexter, K.J., Kai, S., Anandarajah, P.M., Barry, L.P., Wei-Hua, G., O'Dowd, J., Lynch, M., Bradley, A.L., and Donegan, J.F.: 'Two-photon-absorption-based OSNR monitor for NRZ-PSK transmission systems', IEEE Photonics Technol. Lett., 2010, 22, (5), pp. 275-277

7 Guo, W.H., O’Dowd, J., Lynch, M., Bradley, A.L., Donegan, J.F., Barry, L.P., and Kilper, D.C.: 'Two-photon absorption generated by optically amplified signals', Electron. Lett., 2008, 44, (18), pp. 1087-1088

8 O'Dowd, J., Guo, W.H., Lynch, M., Flood, E., Bradley, A.L., and Donegan, J.F.: 'Description of polarisation dependence of two-photon absorption in silicon avalanche photodiodes', Electron. Lett., 2010, 46, (12), pp. 854-856

9 Eliyahu, D.: 'Statistics of Stokes variables for correlated Gaussian fields', Phys. Rev. E, 1994, 50, (3), pp. 2381-2384 